guidelines for reporting trials. In this case, when the light boxes were modified to 100 lux, the disparity in intensity was very obvious and we did not feel that the study would conform to the important double-blind aspect of the design. It would have been very clear to any patient who received the 100 lux box that they had been assigned to the low-intensity arm of the trial. We therefore modified the boxes to administer 2000 lux at $20 \mathrm{~min}$ in the low-intensity arm. The boxes appeared bright, but literature on seasonal affective disorder indicates that this would not be a therapeutic dose within this time frame, whereas 10000 lux at $20 \mathrm{~min}$ would be a therapeutic intensity/dose.

As we stated in the introduction to our study, the primary outcome measure for this trial was seizure control. We have reported these results separately ${ }^{1}$ and that paper is fully referenced in our study. Although it is possible that bright light therapy may result in an increase in seizures for some patients, this was not a statistically significant finding in our previous study and, as yet, the risk remains theoretical. Clinicians will be aware that seizure control should be carefully monitored following the introduction of any new treatment offered to people with epilepsy.

In presenting the results of our study for publication we have sought to provide as clear an account of the data as possible. The results are by no means clear-cut or definitive. However, there are some interesting aspects to the data that suggest that this may not be a dead end in terms of a treatment option for some people with epilepsy. This study stands as a guide for future research. We hope that its limitations, which we fully acknowledge and have set out at length in the Discussion, will serve as a useful guide for future research in this area.

1 Baxendale S, O'Sullivan J, Heaney D. Bright light therapy as an add on treatment for medically intractable epilepsy. Epilepsy Behav 2012; 24: 359-64.

Sallie Baxendale (on behalf of the authors), Department of Neuropsychology (Box 37), National Hospital for Neurology \& Neurosurgery, Queen Square, London WC1N 3BG, UK. Email: s.baxendale@ucl.ac.uk

doi: $10.1192 / \mathrm{bjp} .202 .6 .465 \mathrm{~b}$

\section{Results for behavioural activation are overstated}

The study by Moradveisi et al, ${ }^{1}$ which is applicable to both secondary mental health and primary care, looks at the prospect of using minimally trained staff in delivering behavioural activation against pharmacological intervention in the treatment of severe depression. We would like to highlight the following points for further clarification.

First, an obvious problem of the study was the lack of a placebo arm, which would have lent credibility. As the cultural avoidance of antidepressants in Iran has been highlighted, adding a placebo group would have removed some bias such as paying for medication in the treatment as usual (TAU) group after 3 months and also in the analysis.

Second, sertraline was used at a suboptimal dose and was slowly titrated, against prevailing practice. A meta-analysis shows an optimum dose for sertraline between 100 and $150 \mathrm{mg} /$ day doses below the therapeutic range were significantly less effective, i.e. by $7 \% .^{2}$ Sertraline reached its lowest therapeutic dose of $100 \mathrm{mg}$ at 6 weeks. All drop-outs occurred before the mid-point assessment and only three were as a result of medication side-effects.

Third, there was a significant difference in the amount of attention that participants received in each group. Participants in the behavioural activation group received 50\% more face-toface sessions than the TAU group. The study did not adjust for this in the analysis.
Fourth, last observation carried forward (LOCF) was used in the study. However, $5 \%$ of drop-outs occurred in the behavioural activation group as opposed to a significant 30\% from the TAU group. Last observation carried forward is used frequently in intention-to-treat studies but standard errors and confidence intervals from LOCF underestimate uncertainty. ${ }^{3}$ As there are no strategies for universal use, reasons for the choice of a certain method have to be provided when designing and analysing clinical trials. ${ }^{4}$ Last observation carried forward analysis seems to have favoured the behavioural activation group.

Many other limitations of the study are cited in the paper itself. Significant numbers of participants were recruited via advertisement or word of mouth, which seemed to have attracted more women and perhaps more psychologically minded individuals. It would have been helpful to include these advertisements as a supplement to the paper in order to identify any bias.

Finally, we wondered whether an ethics committee would allow this type of study to go ahead in the UK as it included individuals with severe depression. In England and Wales, before recruitment to a trial, potential participants must be assessed under the Mental Capacity Act 2005; in Scotland, the Adults with Incapacity (Scotland) Act 2000 (para. 72) must be used. ${ }^{5}$ Since the authors of the study state that 'the study's aim was to investigate whether a simple psychological treatment [...] would be a viable alternative to antidepressant medication [. . .] in a non-Western country', we are unsure of an equivalent law in Iran and whether this criterion was met.

1 Moradveisi L, Huibers MJH, Renner F, Arasteh M, Arntz A. Behavioural activation $\mathrm{V}$. antidepressant medication for treating depression in Iran: randomised trial. Br J Psychiatry 2013; 202: 204-11.

2 Bollini P, Pampallona S, Tibaldi G, Kupelnick B, Munizza C. Effectiveness of antidepressants. Meta-analysis of dose-effect relationships in randomised clinical trials. Br J Psychiatry 1999; 174: 297-303.

3 Mallinckrodt C, Clark W, David S. Accounting for dropout bias using mixedeffects models. J Biopharm Stat 2001; 11: 9-21.

4 Unnebrink K, Windeler J. Intention-to-treat: methods for dealing with missing values in clinical trials of progressively deteriorating diseases. Stat Med 2001; 20: $3931-46$.

5 General Medical Council. Consent: Patients and Doctors Making Decisions Together. GMC, 2008.

Mukesh Kripalani, Adult Crisis Resolution \& Intensive Home Treatment, Tees, Esk and Wear Valleys NHS Foundation Trust, Middlesbrough, UK. Email: drmukesh@doctors.org.uk; Muhammad Suleman, Adult Crisis Resolution \& Intensive Home Treatment, Adult Affective Team, Tees, Esk and Wear Valleys NHS Foundation Trust, Middlesbrough, UK

doi: 10.1192/bjp.202.6.466

Authors' reply: We thank Kripalani \& Suleman for their critical remarks. Before addressing them point by point, a general remark is required. Our trial was an effectiveness, not an efficacy, trial. We compared a new treatment previously tested elsewhere (behavioural activation) with treatment as usual (TAU) (antidepressant medication) in Iran. An effectiveness trial aims to assess outcomes in usual care, not to test specific mechanisms, which affects the type of control condition(s). Some criticisms make sense from an efficacy study point of view, not from an effectiveness study point of view. Also of note is that the initial response to TAU was quite good, and that the longer-term response of behavioural activation accounted for its superiority.

We do not see how a placebo arm could have assessed cultural influences on TAU. To study this interesting topic, both a placebo and a natural course condition are needed to see whether placebo in Iran does worse than in other cultures compared with doing nothing. 